\title{
Prevalence of antibodies to Rickettsia conorii in human beings and dogs from Catalonia: a 20-year perspective
}

\author{
E. ESPEJO ${ }^{1 *}$, M. ANDRÉS ${ }^{1}$, J. PÉREZ ${ }^{2}$, J. PRAT ${ }^{3}$, C. GUERRERO ${ }^{4}$, \\ M. T. MUÑOZ ${ }^{4}$, M. D. ALEGRE ${ }^{2}$, J. LITE $^{2}$ AND F. BELLA ${ }^{1}$ \\ 1 Infectious Diseases Unit, Internal Medicine Service, Hospital de Terrassa, Terrassa, Spain \\ ${ }^{2}$ Microbiology Laboratory, Catlab, Terrassa, Spain \\ ${ }^{3}$ Hospital Veterinari J. Prat Morera, Terrassa, Spain \\ ${ }^{4}$ Internal Medicine Service, Hospital de Terrassa, Terrassa, Spain
}

Received 13 May 2015; Final revision 28 December 2015; Accepted 29 January 2016; first published online 22 February 2016

\section{SUMMARY}

The incidence of Mediterranean spotted fever (MSF) in Catalonia (Spain) has decreased in the last two decades. The prevalence of antibodies to Rickettsia conorii in human beings and dogs in the region of Vallès Occidental (Catalonia) was assessed by indirect immunofluorescence, and the results compared with those obtained in a similar study from 1987. Nineteen (5.0\%) out of 383 human serum samples had antibodies to $R$. conorii. This seroprevalence was significantly lower $(11 \cdot 5 \%)(P=0 \cdot 003)$ than that recorded in the 1987 survey. Forty-two out (42.0\%) of 100 canine serum samples had antibodies to $R$. conorii. A high proportion of the studied dogs $(91.0 \%)$ were receiving anti-tick treatment, mainly with permethrin-imidacloprid spot-on (Advantix, Bayer, Germany). The current canine seroprevalence was not significantly different from that recorded in the 1987 survey (36.9\%). In conclusion, this study shows a significant decrease in the prevalence of antibodies to $R$. conorii in the human population of Catalonia in the last 20 years, which corresponds with a decrease in the number of cases of MSF. We suggest that the widespread use of anti-tick treatment in dogs could limit the introduction of ticks to humans due to a reduction of infestation duration in dogs, thus contributing to the decrease in MSF incidence.

Key words: Dogs, Mediterranean spotted fever, Rickettsia conorii, seroprevalence, tick-borne disease.

\section{INTRODUCTION}

Mediterranean spotted fever (MSF) is a tick-borne infection caused by Rickettsia $(R$.) conorii and is endemic in the Mediterranean basin [1]. The habitual reservoir and dominant vector is the dog tick, Rhipicephalus (Rh.) sanguineus [2, 3]. Dogs play an important role in MSF epidemiology, carrying infected

\footnotetext{
* Author for correspondence: Dr E. Espejo, Infectious Diseases Unit, Hospital de Terrassa, Consorci Sanitari de Terrassa, Ctra. Torrebonica s/n, 08227 Terrassa, Spain.

(Email: 12088eea@comb.cat)
}

ticks close to human beings. In our experience, between $85 \%$ and $91 \%$ of patients with MSF report contact with dogs $[1,4]$.

MSF seems to have cyclical variation in incidence, but the causes of these variations are unknown [5]. In Catalonia, as in Italy and southern France, the incidence of the disease increased sharply in the 1980s [6-8]. By contrast, in the last 15 years we have observed a significant reduction in the incidence of the disease.

Seroprevalence in humans and especially in dogs has been considered a good epidemiological marker of $R$. conorii infection [2, 9-11]. In the region of 
Vallès Occidental (Catalonia, Spain), we performed a seroepidemiological study in humans and dogs in 1987, which documented a wide diffusion of $R$. conorii [12].

The aim of this study was to determine the current prevalence of antibodies to $R$. conorii in humans and dogs in Catalonia and to compare it with that obtained in 1987.

\section{MATERIALS AND METHODS}

The Hospital de Terrassa is located in the region of Vallès Occidental (Catalonia) and serves a population of about 200000 . From 1987, all cases of MSF diagnosed at our institution were prospectively recorded in a database. MSF is a notifiable disease in Catalonia. The reported incidence rate of MSF in Catalonia (cases/100 000 persons per year) was obtained from the Health Department, Government of Catalonia.

The methodology for obtaining samples of human and canine serum was the same as that used in the 1987 study [12].

\section{Human serum samples}

Between May 2008 and July 2009, human serum samples from 383 individuals over 14 years undergoing routine medical examination at the Hospital de Terrassa were collected. The selection of subjects was performed according to the gender and age frequency distribution for the area, as performed in the 1987 survey. In each case a questionnaire was completed, in which the following variables were recorded: age, sex, occupation, place of residence, contact with dogs, contact with other animals, and outdoor activities in the last 10 years. Veterinarian, cattle rancher, and farmer were considered risky occupations. Hiking, horticulture, and hunting were considered risky practices. Patients with an active infectious disease, immune abnormalities or a history of MSF were excluded. The study population was stratified by age ( $<40$ years, $40-70$ years, $>70$ years). A sample size of 383 was calculated to detect a statistically significant difference of $40 \%$ in the prevalence between the current study and the 1987 study (11.5\%), with $80 \%$ power and 5\% significance level.

\section{Dog serum samples}

During the summer months of 2012 and 2013, serum samples were collected from 100 dogs attending a veterinary clinic located in Terrassa (Catalonia). The veterinary clinic was one of the two that had participated in the 1987 study. Dogs requiring a blood test were selected following the procedure of that study. All dogs were privately owned and information about breed, age, habitat, presence of ticks and use of anti-tick treatments was obtained in each case. A sample size of 100 was calculated to detect a statistically significant difference of $33 \%$ in the prevalence between the current study and the 1987 study (36.8\%), with $80 \%$ power and $5 \%$ significance level.

\section{Serological technique}

The serum samples were assayed by indirect immunofluorescence using commercially available antigens obtained from a Vero cell culture infected with R. conorii. For human sera, determination of antibodies was carried out using anti-human IgG globulin labelled with fluorescein (Rickettsia conorii IgG IFI PRICOG; Vircell, Spain). For canine sera, a fluorescence-labelled anti-dog IgG conjugate (MegaScreen FLUO RICKETTSIA, Eurovet Veterinaria, Spain) was used. The procedure was conducted according to the manufacturer's instructions. Twofold dilutions, beginning at 1:40, were made for each serum. Titres of $\geqslant 1: 40$ were considered positive.

\section{Statistical analysis}

Descriptive analysis was performed using percentages with $95 \%$ confidence intervals (CIs) for categorical variables and means with standard deviation (s.D.) for continuous variables. Statistical comparison of categorical variables was performed by $\chi^{2}$ test, or Fisher's exact test for $2 \times 2$ tables where any cell contained $<5$ cases. Statistical comparison of continuous variables was performed with the Student's $t$ test and, when the assumptions of $t$ test were not met, with the Mann-Whitney $U$ test. $P \leqslant 0.05$ was considered statistically significant. Statistical analysis was performed using Epidat v. 3.1 (epidat.software. informer.com/3.1).

\section{Ethical standards}

The institutional review board of the Hospital de Terrassa approved the study and written informed consent was obtained from all participants in the study. Informed consent was also obtained from each dogowner and all applicable international and national guidelines for the care and use of animals were followed. 


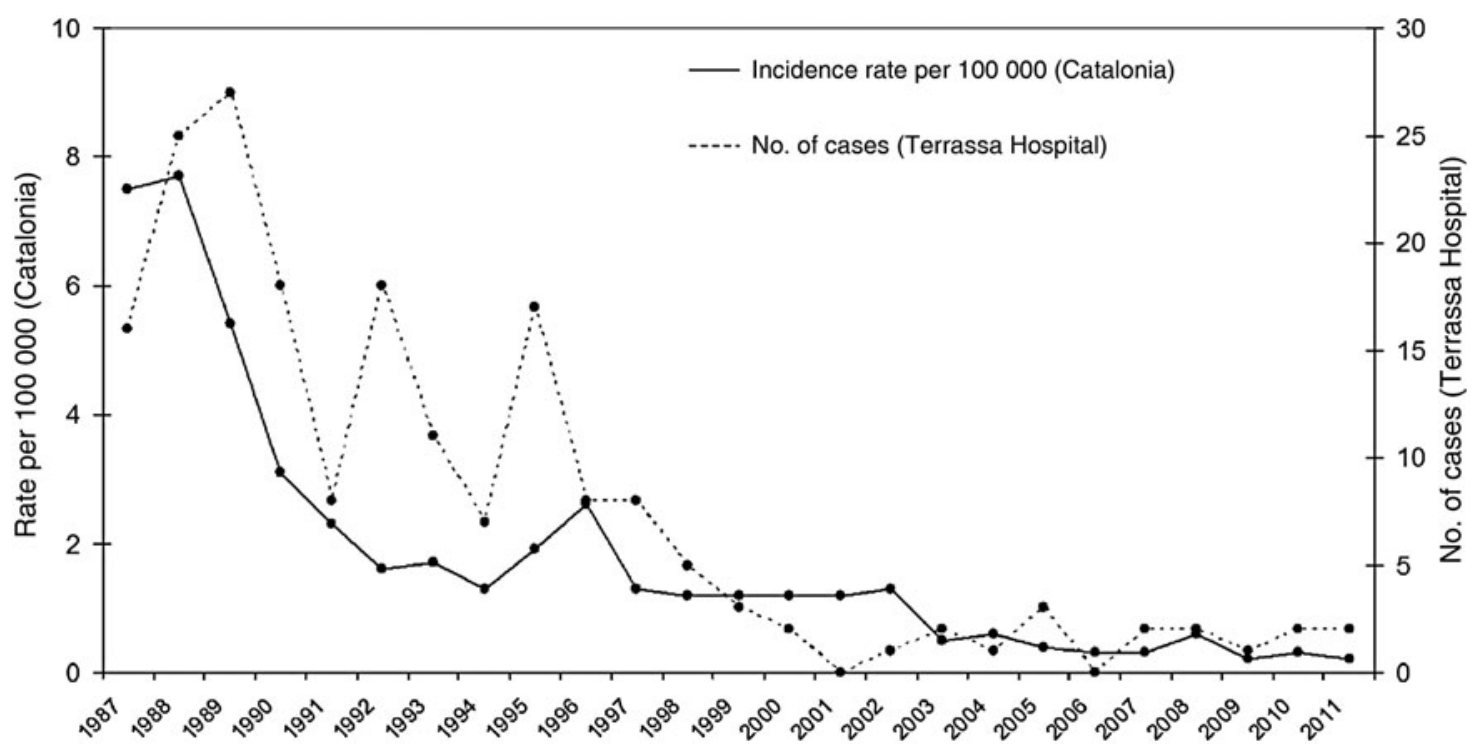

Fig. 1. Annual reported incidence rates of Mediterranean spotted fever in Catalonia (cases/100 000 persons per year), and annual number of cases diagnosed at Hospital de Terrassa, 1987-2011.

\section{RESULTS}

Figure 1 shows the evolution of the annual incidence of MSF reported to the notifiable diseases surveillance system in Catalonia, and the evolution of the number of MSF cases diagnosed at our institution from 1987 to 2011. A significant decrease in the incidence of the disease over time can be seen.

\section{Human population}

Of the 383 subjects studied, $181(47 \cdot 3 \%)$ were men and $202(52 \cdot 7 \%)$ were women. Ages ranged from 15 to 89 years, with a mean age of $46 \cdot 4$ years (s.D. $=18 \cdot 1$ ). A total of $312(81.5 \%)$ subjects lived in urban areas and $71(18.5 \%)$ lived in rural or semi-rural areas. A total of $111(29 \cdot 0 \%)$ subjects participated in outdoor activities and $25(6 \cdot 5 \%)$ were involved with horticultural practices. The distribution of these variables was not significantly different from that observed in the sample for the study conducted in 1987.

Overall, 19 human sera $(5 \cdot 0 \%, 95 \%$ CI $2 \cdot 8-7 \cdot 1)$ had antibodies to $R$. conorii. The titre of antibodies to $R$. conorii was 1:40 in three cases, 1:80 in 10 cases, 1:160 in five cases and 1:320 in one case. Table 1 shows the seroprevalence according to sex, age, habitat and outdoor activities. Seropositivity rate was higher in men than in women but the difference was not statistically significant $(P=0.058)$. There was strong evidence to suggest a difference in the mean age of seropositive subjects ( 58.9 years, S.D. $=16.5)$ and that of seronegative subjects ( 45.7 years, S.D. $=18 \cdot 0)(P=0 \cdot 02)$. A significant correlation between seropositivity rate and occupation, contact with animals or risky practices was not detected.

The seroprevalence recorded in this study $(5 \cdot 0 \%)$ was significantly lower $(P=0.003)$ than that recorded in the 1987 survey $(11 \cdot 5 \%)$.

\section{Canine population}

Of the 100 dogs studied, 71 lived in an urban area and 29 in a rural or semi-rural area. Fifty-six dogs lived in an apartment and 44 in a house. Sixty-six dogs lived indoors and 34 outdoors. Regarding anti-tick treatment, 88 dogs were treated with permethrinimidacloprid spot-on (Advantix, Bayer, Germany), 71 dogs were treated with a deltamethrin collar and 68 dogs were treated with both. Only nine dogs did not receive any anti-tick treatment.

Overall, 42 canine serum samples $(42 \cdot 0 \%, 95 \%$ CI $32 \cdot 3-51 \cdot 7)$ had antibodies to $R$. conorii, The titre of antibodies to $R$. conorii was $1: 40$ in 13 dogs, $1: 80$ in nine dogs, 1:160 in 12 dogs, 1:320 in four dogs, 1:640 in three dogs, and 1:1280 in one dog.

Table 2 shows the seroprevalence according to breed and habitat of the studied dogs. No significant differences were observed. Table 3 shows the seroprevalence regarding anti-tick treatment. There was weak evidence to suggest that dogs treated with permethrin-imidacloprid spot-on had a lower seroprevalence than those not treated with this method $(P=0 \cdot 06)$. Attached ticks were observed in five dogs, three of which had antibodies to $R$. conorii. 
Table 1. Seroprevalence to Rickettsia conorii in the human population according to several factors

\begin{tabular}{|c|c|c|c|c|}
\hline & \multirow{2}{*}{$\begin{array}{l}\text { No. of } \\
\text { subjects }\end{array}$} & \multicolumn{3}{|c|}{ Seropositivity } \\
\hline & & No. & $\%$ & $P$ \\
\hline \multicolumn{5}{|l|}{ Age (years) } \\
\hline$<40$ & 176 & 3 & $1 \cdot 7$ & $<0.003$ \\
\hline $40-70$ & 162 & 10 & $6 \cdot 8$ & \\
\hline$>70$ & 45 & 6 & $13 \cdot 3$ & \\
\hline \multicolumn{5}{|l|}{ Sex } \\
\hline Male & 181 & 13 & $7 \cdot 2$ & 0.058 \\
\hline Female & 202 & 6 & $3 \cdot 0$ & \\
\hline \multicolumn{5}{|l|}{ Residential area* } \\
\hline Urban & 312 & 15 & $4 \cdot 8$ & 0.763 \\
\hline Rural or semi-rural & 71 & 4 & $5 \cdot 6$ & \\
\hline \multicolumn{5}{|l|}{ Other factors } \\
\hline Risky occupation $\dagger$ & 13 & 1 & $7 \cdot 6$ & $0 \cdot 489$ \\
\hline No risky occupation & 370 & 18 & $4 \cdot 9$ & \\
\hline Contact with dogs & 152 & 8 & $5 \cdot 2$ & $0 \cdot 825$ \\
\hline No contact with dogs & 231 & 11 & $4 \cdot 8$ & \\
\hline Outdoor activities & 111 & 6 & $5 \cdot 4$ & 0.797 \\
\hline No outdoor activities & 272 & 13 & $4 \cdot 8$ & \\
\hline Practice of horticulture & 25 & 3 & $12 \cdot 0$ & $0 \cdot 118$ \\
\hline $\begin{array}{l}\text { No practice of } \\
\text { horticulture }\end{array}$ & 358 & 16 & $4 \cdot 5$ & \\
\hline
\end{tabular}

* Defined by number of inhabitants of municipalities: rural $<5000$, semi-rural 5000-50000, urban $>50000$.

$\uparrow$ Veterinarian, breeder or farmer.

There was no significant difference between the current seroprevalence $(42.0 \%)$ and that recorded in the 1987 survey $(36 \cdot 9 \%)(P=0 \cdot 45)$.

\section{DISCUSSION}

The causes of the cyclic variations of MSF incidence are unknown [2, 5]. Various factors have been suggested to explain the increase in incidence observed in the early 1980s, such as an increased number of ticks, increased human contact with the habitat of infected ticks or ecological changes in the outskirts of large cities resulting from the movement of rural sources of infected ticks to suburban zones [5]. Climatic changes, such as increased temperature and decreased rainfall, are suspected to play a role in tick activity and MSF prevalence [13-16]. Another factor could be a shift in the anti-rickettsial effectiveness of the most used antibiotics as empirical therapy for patients with fever of unknown origin [5]; thus, in the late 1970s and early 1980s, decreased use of doxycycline and increased use of amoxicillin-clavulanate, which lacks anti-rickettsial activity, coincided with an increase in MSF incidence.
Table 2. Seroprevalence to Rickettsia conorii in the canine population according to breed and habitat

\begin{tabular}{|c|c|c|c|}
\hline & \multirow{2}{*}{$\begin{array}{l}\text { No. of } \\
\text { dogs }\end{array}$} & \multicolumn{2}{|c|}{ Seropositivity* } \\
\hline & & No. & $\%$ \\
\hline \multicolumn{4}{|l|}{ Breed } \\
\hline German shepherd & 18 & 8 & $44 \cdot 4$ \\
\hline Boxer & 9 & 3 & $33 \cdot 3$ \\
\hline Yorkshire terrier & 8 & 5 & $62 \cdot 5$ \\
\hline Labrador terrier & 6 & 3 & $50 \cdot 0$ \\
\hline Schnauzer & 5 & 0 & 0 \\
\hline Golden retriever & 5 & 2 & $40 \cdot 0$ \\
\hline Poodle & 4 & 2 & $50 \cdot 0$ \\
\hline $\begin{array}{l}\text { West Highland white } \\
\text { terrier }\end{array}$ & 4 & 1 & $25 \cdot 0$ \\
\hline Shi-tzu & 3 & 0 & 0 \\
\hline Other breed & 30 & 13 & $40 \cdot 9$ \\
\hline $\begin{array}{l}\text { Cross-breed or } \\
\text { undefined breed }\end{array}$ & 8 & 5 & $62 \cdot 5$ \\
\hline \multicolumn{4}{|l|}{ Residential area $\dagger$} \\
\hline Urban & 71 & 29 & $40 \cdot 8$ \\
\hline Rural or semi-rural & 29 & 13 & $44 \cdot 8$ \\
\hline \multicolumn{4}{|l|}{ Dog accommodation } \\
\hline Inside the dwelling & 66 & 26 & $39 \cdot 4$ \\
\hline Outdoors & 34 & 16 & $47 \cdot 1$ \\
\hline
\end{tabular}

* No statistically significant differences related to breed, habitat or dog accommodation were observed.

$\dagger$ Defined by number of inhabitants of municipalities: rural $<5000$, semi-rural 5000-50000, urban $>50000$.

In Catalonia, where MSF is a notifiable disease, there has been a marked decrease in the incidence reported in the last 15 years. While it is likely that there is underreporting of the disease, there is no reason to believe that underreporting is greater now than it was 20 years ago. Moreover, the evolution of the number of cases as seen in the prospective registry at our hospital is consistent with this decrease, as noted also by other authors since 1998 [17].

IgG antibodies to $R$. conorii may remain detectable for a long time [18]. Thus, between 5 and 10 years after suffering from MSF, more than half of patients have IgG titres to $R$. conorii $\geqslant 1: 40$ [12]. Therefore, the seroprevalence studies in the human population may reflect changes in incidence of the disease only if performed with a time separation of many years, as is the case in this study. Our finding of a significant decrease in the prevalence of antibodies to $R$. conorii in the human population in our area, over a period of 20 years, seems to confirm a lower exposure of this population to $R$. conorii.

While in our area more than $85 \%$ of patients with MSF report contact with dogs $[1,4]$, it is noteworthy 
Table 3. Seroprevalence to Rickettsia conorii in the canine population according to anti-tick treatment

\begin{tabular}{|c|c|c|c|c|}
\hline \multirow[b]{2}{*}{ Anti-tick treatment } & \multirow{2}{*}{$\begin{array}{l}\text { No. of } \\
\text { dogs }\end{array}$} & \multicolumn{3}{|c|}{ Seropositivity } \\
\hline & & No. & $\%$ & $P$ \\
\hline $\begin{array}{l}\text { Only permethrin-imidacloprid } \\
\text { spot-on }\end{array}$ & 20 & 9 & $45 \cdot 5$ & $0 \cdot 279$ \\
\hline Only deltamethrin collar & 3 & 2 & $66 \cdot 7$ & \\
\hline $\begin{array}{l}\text { Permethrin-imidacloprid } \\
\text { spot-on plus deltamethrin } \\
\text { collar }\end{array}$ & 68 & 25 & $36 \cdot 8$ & \\
\hline No anti-tick treatment & 9 & 6 & $66 \cdot 7$ & \\
\hline $\begin{array}{l}\text { Permethrin-imidacloprid } \\
\text { spot-on* }\end{array}$ & 88 & 34 & $38 \cdot 6$ & $0 \cdot 064$ \\
\hline $\begin{array}{l}\text { No permethrin-imidacloprid } \\
\text { spot-on* }\end{array}$ & 12 & 8 & $66 \cdot 6$ & \\
\hline Deltamethrin collar $\dagger$ & 71 & 27 & $38 \cdot 0$ & $0 \cdot 207$ \\
\hline No deltamethrin collar $\dagger$ & 29 & 15 & $51 \cdot 7$ & \\
\hline
\end{tabular}

* With or without deltamethrin collar.

$\dagger$ With or without permethrin-imidacloprid spot-on.

that the contact with dogs did not represent an increase in seropositivity in this study. It is possible that some individuals had been in contact with dogs for $>10$ years before, since this is the time period that was investigated in this study. Moreover, it has been suggested that, in addition to unnoticed tick bites, inhalation or ingestion of dry products containing antigenically active rickettsial material could explain the seropositivity in some cases [10, 12].

A higher prevalence of antibodies to $R$. conorii with advancing age was also observed in our previous study [12]. It probably reflects the accumulated exposure over the years because of the prolonged persistence of antibodies.

Unlike what occurs in humans, the duration of the immune response to $R$. conorii in dogs is relatively short [19], with a much higher seroprevalence in summer than in winter and spring $[11,12]$. Therefore, it is recommended that prevalence studies in dogs be performed during summer [19], as was the case in both our studies. Our finding that canine seroprevalence has not changed significantly in the last 20 years with regard to $R$. conorii seems to indicate that the contact of dogs with this bacterium remains stable.

The very frequent use of anti-tick treatment in the studied dogs is noteworthy. In fact, almost all dogs were treated with permethrin-imidacloprid spot-on and the majority also wore a deltamethrin collar during summer months. Various veterinarians confirmed that a large proportion of dogs in our area receive this preventive treatment. In the 1980s, the spot-on treatment had not yet been introduced and the use of a deltamethrin collar was most uncommon. Experimentally it has been demonstrated that treatment with permethrin spot-on reduces the infestation of dogs by Rh. sanguineus, but it does not suppress infestation completely [20]. However, Lüssenhop et al. demonstrated that in dogs treated with permethrinimidacloprid spot-on, most ticks were dead $6 \mathrm{~h}$ after experimental infestation with ticks of the genus Dermacentor reticulatus [21]. In fact, many dogowners who use the spot-on treatment report that it is not uncommon to find ticks attached to the dog, but that usually these ticks are dead. Thus, it appears that these treatments do not completely eliminate the parasitic infestation (and therefore the possibility of infection by $R$. conorii, if the tick is infected) but significantly reduce its duration, such that the role of the dog as a vehicle that brings ticks into close contact with humans is significantly reduced. This could explain our findings of a stable seroprevalence in the dogs, and a decrease in the incidence of MSF and the seroprevalence in the human population. Of course, other factors might have contributed to the decrease in MSF incidence, such as the greater empirical use in recent years of macrolides and fluoroquinolones with anti-rickettsial activity.

The main limitation of this study is that only immunofluorescence was used to detect the presence of antibodies. Several studies have shown that use of Western blotting increases the specificity of the positive results, reducing the number of false positives due to crossreactivity with species of Rickettsia other than $R$. conorii [22]. However, taking into account that the main objective of the study was to compare the current seroprevalence with that obtained in 1987, we think that it was appropriate to reproduce as closely as possible the methodology used in that study, both with regards to obtaining samples and to the processing of the sera. Another limitation could be the time difference between the collection of human and canine sera, due to the fact that the study in dogs was designed and conducted after the completion of the study in humans. However, considering that the immune response to $R$. conorii persists for a long time in humans, the 4-year interval between the collection of the human sera and the canine sera is not significant with regard to the prevalence of antibodies to $R$. conorii.

In conclusion, our study shows a significant decrease in the prevalence of antibodies to $R$. conorii in the human population of Catalonia in the last 20 
years, which corresponds to a decrease in the number of MSF cases, whereas the seroprevalence in the canine population has remained the same. We suggest that the widespread use of permethrin-imidacloprid spot-on treatment in dogs could limit the introduction of ticks to humans due to the resulting reduced infestation time in dogs, thus contributing to the decrease in MSF incidence.

\section{ACKNOWLEDGEMENTS}

The authors thank Maria Rosa Sala (Epidemiological Surveillance Unit, Health Department, Generalitat de Catalunya) for her assistance in obtaining the annual incidence rates of Mediterranean spotted fever in Catalonia. The authors also thank Míriam Nieto, Carles Prat, Francesc Darnacolleta and Alex De Jorge for their assistance in obtaining canine sera.

This work was supported in part by a grant from the Fundació Joan Costa Roma, Terrassa (2006-03).

\section{DECLARATION OF INTEREST}

None.

\section{REFERENCES}

1. Font-Creus B, et al. Mediterranean spotted fever: a cooperative study of 227 cases. Reviews of Infectious Diseases 1985; 7: 635-642.

2. Rovery C, Raoult D. Mediterranean spotted fever. Infectious Disease Clinics of North America 2008; 22: 515-530.

3. Gilot B, et al. Relationships between the Rhipicephalus sanguineus complex ecology and Mediterranean spotted fever epidemiology in France. European Journal of Epidemiology 1990; 6: 357-362.

4. Font Creus B, et al. Mediterranean boutonneuse fever. Study of 246 cases. Medicina Clínica 1991; 96: 121-125.

5. Rovery C, Brouqui P, Raoult D. Questions on Mediterranean spotted fever a century after its discovery. Emerging Infectious Diseases 2008; 14: 1360-1367.

6. Segura-Porta F, et al. New trends in Mediterranean spotted fever. European Journal of Epidemiology 1989; 5: 438-443.

7. Mansueto S, Tringali G, Walker DH. Widespread, simultaneous increase in the incidence of spotted fever group rickettsioses. Journal of Infectious Diseases 1986; 154: 539-540.
8. Raoult D, et al. Mediterranean boutonneuse fever. Apropos of 154 recent cases. Annales de Dermatologie et de Vénéréologie 1983; 110: 909-914.

9. Mannelli A, et al. Associations between dogs that were serologically positive for Rickettsia conorii relative to the residences of two human cases of Mediterranean spotted fever in Piemonte (Italy). Preventive Veterinary Medicine 2003: 60: 13-26.

10. Herrero-Herrero JI, et al. Mediterranean spotted fever in Salamanca, Spain. Epidemiological study in patients and serosurvey in animals and healthy human population. Acta Tropica 1989; 46: 335-350.

11. Ortuño A, et al. The dog as an epidemiological marker of Rickettsia conorii infection. Clinical Microbiology and Infection 2009; 15 (Suppl. 2): 241-242.

12. Espejo-Arenas E, et al. Seroepidemiological survey of Mediterranean spotted fever in an endemic area ('Vallés Occidental', Barcelona, Spain). Tropical and Geographical Medicine 1990; 42: 212-216.

13. Espejo-Arenas E, et al. Climatic factors in resurgence of Mediterranean spotted fever. Lancet 1986; 1: 1333.

14. Parola P, et al. Warmer weather linked to tick attack and emergence of severe ricketssioses. PLOS Neglected Tropical Diseases 2008; 2: e338.

15. De Sousa R, et al. Boutonneuse fever and climate variability. Annals of the New York Academy of Sciences 2006; 1078: 162-169.

16. Estrada-Peña A, Ayllón N, De la Fuente J. Impact of climate trends on tick-borne pathogen transmission. Frontiers in Physiology 2012; 3: 64.

17. Segura-Porta F, et al. Prevalence of antibodies to spotted fever group rickettsiae in human beings and dogs from an endemic area of Mediterranean spotted fever in Catalonia, Spain. European Journal of Epidemiology 1998; 14: 395-398.

18. Mansueto S, et al. Persistence of antibodies to Rickettsia conorii after an acute attack of boutonneuse fever. Journal of Infectious Diseases 1985; 151: 377.

19. Espejo E, et al. Antibodies to Rickettsia conorii in dogs: seasonal differences. European Journal of Epidemiology 1993; 9: 344-346.

20. Estrada-Peña A, Venzal Bianchi JM. Efficacy of several anti-tick treatments to prevent the transmission of Rickettsia conorii under natural conditions. Annals of the New York Academy of Sciences 2006; 1078: 506-508.

21. Lüssenhop J, et al. Dynamics of distribution and efficacy of different spot-on permethrin formulations in dogs artificially infested with Dermacentor reticulatus. Parasites \& Vectors 2011; 4: 45.

22. Babalis T, et al. Rickettsia conorii in Greece: comparison of a microimmunofluorescence assay and Western blotting for seroepidemiology. American Journal of Tropical Medicine and Hygiene 1993; 48: 784-792. 